\title{
ALTERIDAD, NÚMERO Y REFLEXIÓN TRINITARIA EN NICOLÁS DE CUSA
}

\section{ALTERITY, NUMBER AND TRINITARIAN REFLECTION IN NICHOLAS OF CUSA}

\author{
RODRIGO NÚÑEZ POBLETE* \\ Dr. en Filosofía \\ Universidad Católica del Maule - Talca - Chile \\ Centro de Investigación en Religión y Sociedad (CIRS UCM)
}

Artículo recibido el II de agosto de 2020; aceptado el o9 de octubre de 2020.

"rnunezp@ucm.cl

https://orcid.org/oooo-oooI-77I7-980I

Cómo citar este artículo:

R. Núñez. P. (2020). "Alteridad, número y reflexión trinitaria en Nicolás de Cusa" en Palabra y Razón. Revista de Teología, Filosofía y Ciencias de la Religión. № I8 Diciembre 2020, pp 69-88 https:// doi.org/I0.29035/pyr.18.69 


\section{RESUMEN}

El presente artículo examina la negatividad concomitante a la alteridad en el pensamiento cusano y la posibilidad de reconocer las huellas del principio unitrino en lo finito. Para lograr lo anterior, (i) se analiza la constatación negativa de la alteridad en la metafísica de la finitud vinculada a la contingencia, luego (ii) se describe la función articuladora que cumple el número en la comprensión de la finitud como multiplicidad creada por el primer principio de todo, toda vez que esta creación es un despliegue de la potencia del número divino. Finalmente, (ii) se analiza el lugar que tiene la alteridad en la argumentación trinitaria del libro primero de De docta ignorantia, con lo cual, se mostrará cómo la alteridad abre un espacio para una consideración del primer principio que, si bien excluye de sí toda alteridad, a la vez, funda la totalidad de lo otro. Palabras
Cusa
ABSTRACT

Palabras claves: Alteridad / número / especulación trinitaria / Nicolás de

This article addresses the concomitant negativity regarding alterity in cusanian thought and the possibility of recognizing the traces of the unitrinity principle in the finite. To achieve this, (i) it is analysed the negative verification of alterity in the metaphysics of finitude linked to contingency; (ii) it is described the articulating function that the number plays in the understanding of finitude as created multiplicity by the first principle, God, insofar as this creation is a deployment of the divine number's potency; finally, (ii) it is analysed the place that alterity has in the trinitarian argumentation of the first book of De docta ignorantia, with which it will be shown how alterity opens up a space for a consideration of the first principle that, although it excludes from itself all alterity, at the same time, grounds the totality.

Keywords: Alterity / number / trinitarian speculation / Nicholas of Cusa 


\section{I) Introducción}

La alteridad es un fenómeno complejo por su carácter difuso e irreductible que atrae la atención de los filósofos en distintos momentos de la historia del pensamiento. Se trata de una temática crucial que se vincula con la cuestión de la distinción del individuo cuya singularidad lo separa de "lo otro" y con el asunto de las múltiples oposiciones que constituyen la diferencia en lo finito.

En la tradición filosófica los orígenes de la temática de la alteridad se pueden remontar al Sofista de Platón y su tratamiento del binomio mismidad-alteridad como géneros ${ }^{2}$ y a los desarrollos posteriores del neoplatonismo (plotiniano y procleano) que reinterpretan pasajes del Parménides de Platón conduciendo a una creciente priorización de la negación por sobre la afirmación. En ese marco, se plantea que, si lo Uno no se opone a lo múltiple, entonces no es dable predicar de él ni mismidad ni alteridad, sino la negación de aquellos.

Numerosos estudios ${ }^{3}$ han indagado cómo estás tradiciones confluyen en el pensamiento cusano de modo original a través de las relaciones que habría establecido entre el Areopagita y Proclo y que sientan las bases para la interpretación en los escritos cusanos tardíos, de términos como aliud y non-aliud en el sentido de significar los vocablos de tal manera que el intelecto pueda acceder a aquello que es imposible para la razón discursiva, a saber, poder entenderlos más

I Cf. NICOLÁS DE CUSA, De venatione sapientiae XXII (h. XII, n 65): "Nam cum unum non sit aliud quam unum, singulare videtur, quia in se indivisum et ab alio divisum". Las obras cusanas se citan de acuerdo a la edición crítica, donde h: Nicolai de Cusa, Opera Omnia, Iussu et Auctoritate Academiae Litterarum Heidelbergensis, Leipzig, 1932 ss., indicando en cada caso el volumen y número de parágrafo.

2 Cf. PLATÓN, Sofista 254 d-555d; C. D’AMICO, “Unum neque idem neque alterum: novedad y tradición en las nociones cusanas de identidad y alteridad” en J. M. MACHETTA - C. D’AMICO (coord.) Nicolás de Cusa: identidad y alteridad. Pensamiento y diálogo. Biblos: Buenos Aires, 2010, pp. 249-26o, p. 250.

3 Cf. C. D' AMICO, "Alteridad y participación" en NICOLÁS DE CUSA, Acerca de lo no-otro o de la definición que todo lo define, Nuevo texto crítico original (edición bilingüe), J. M. MACHETTA (trad.), C. D'AMICO, M. D'ASCENZO, A. EISENKOF, J. GONZÁLEZ RÍOS, C. RUSCONI y H. SCHWAETZER (preparación del texto crítico y notas complementarias), Biblos: Buenos Aires, 2008. pp. 286-295. W. BEIERWALTES, “'Centrum tocius vite.' Zur Bedeutung von Proklos' «Theologia Platonis» im Denken des Cusanus" en Proclania. Spätantikes Denken und seine Spuren, Vittorio Klostermann: Frankfurt am Main 2007, pp. 19I-2I4; W. BEIERWALTES, "Der verborgene Gott. Cusanus und Dionysius" en Platonismus im Christentum, Vittorio Kostermann: Frankfurt am Main, 200I, pp. I30-I71; W. BEIERWALTES, "Nicolaus Cusanus: Innovation durch Einsicht aus der Überlieferung. Paradigmatisch gezeigt aus seinem Denken des Einen" en: Proclania. Spätantikes Denken und seine Spuren, Klostermann: Frankfurt am Main, 2007, pp. I65-I90; W. BEIERWALTES, "Cusanus und Proklos zum neuplatonischen Ursprung des non aliud" en Niccolo Cusano agli inizi del mondo moderno, Sansoni: Firenze, I970, I37-I4O; E. WYLLER, "Nicolaus Cusanus De non aliud und Platons Dialog Parmenides”, Studia Platonica, Grüner: Amsterdam, 1974, pp. 239-25I, entre otros. 
allá de la disyunción de los opuestos y, al mismo tiempo, anterior a la disyunción entre afirmación y negación ${ }^{4}$. Esta sería la perspectiva más precisa para entender la negación que Nicolás establece de la alteridad en la unidad absoluta al utilizar los términos aludidos de non-aluid de manera enigmágica, es decir, en el sentido de una oppositio oppositorum sine oppositione 5 .

En el nivel ontológico, por otro lado, en el pensar cusano la alteridad queda referida exclusivamente a lo finito en su condición de explicatio de la unidad. Esta idea la encontramos en la obra De coniecturis, donde la alteridad parece ser, además, un principio de distinción dentro de la participación en la unidad. Ahora bien, Nicolás explícitamente niega la alteridad, además, respecto de la primera unidad, la cual complica (complicatio) todolo existente. Pero esto no significa que el primer principio se confunda con lo finito. El descenso de la unidad absoluta en la unidad del universo, la que a su vez solo subsiste en la unidad de individuos, redunda en una dependencia ontológica que hace que Dios no sea un "otro" al modo de la alteridad finita. Todo ser finito y su singularidad surgen de él sin ningún otro condicionamiento, por lo tanto, la alteridad es, en ese marco, más bien nada. La alteridad sólo tiene sentido en el ámbito de lo finito, donde la condición de la singularidad implica la ineludible privación dada por la delimitación de lo otro. ¿Dónde queda, entonces, el sentido de la distinción como explicatio de la identidad, si la condición de esa distinción pareciera más bien ser nada?

Si ampliamos el contexto de este primer nivel ontológico, debemos mencionar la idea de la inenarrable presencia de lo múltiple en la unidad infinita al modo de la complicatio. Todo lo múltiple es en la unidad, pero sin alteridad. En la idea de la posterioridad de la alteridad respecto de la unidad, o desde la otra dirección, en la idea de la prioridad de la unidad, Nicolás integra una serie de razonamientos que vinculan la alteritas con la mutabilitas $^{6}$, la inaequalitas ${ }^{7}$, la divisio $^{8}$. Las tres nociones asociadas a la

4 Cf. C. D'AMICO, "Unum neque idem neque alterum: novedad y tradición en las nociones cusanas...", pp. 252-254.

5 El presente trabajo se concentra en la terminología cusana ligada a la alteritas, dejando fuera del análisis la asociada a término latino aliud. Sobre dicho término y su negación ver al trabajo de C. D' AMICO, “Alteridad y participación...”, pp. 286-295.

6 Cf. NICOLÁS DE CUSA, De docta ignorantia I (h. I, n. I8): "Id, quod omnem alteritatem praecedit, aeternum esse nemo dubitat. Alteritas namque idem est quod mutabilitas; sed omne, quod mutabilitatem naturaliter praecedit, immutabile est; quare aeternum".

7 Cf. NICOLÁS DE CUSA, De docta ignorantia I (h. I, n. I9): "Sed inaequalitas et alteritas simul sunt natura; ubi enim inaequalitas, ibidem necessario alteritas, et e converso. Inter duo namque ad minus erit alteritas. Illa vero ad unum illorum duplicitatem facient; quare erit inaequalitas. Alteritas ergo et inaequalitas simul erunt natura, praesertim cum binarius prima sit alteritas et prima inaequalitas. Sed probatum est aequalitatem praecedere natura inaequalitatem, quare et alteritatem; aequalitas ergo aeterna".

8 Cf. NICOLÁS DE CUSA, De docta ignorantia I (h. I, n. 20): "Sed divisio et alteritas simul sunt 
alteridad surgen en la argumentación que Nicolás ofrece para explicar que tanto la unidad, la igualdad de la unidad y la conexión de ambos son eternas, es decir, la uni-trinidad divina. Ahora bien, en estos argumentos la unidad y la alteridad no están pensados en oposición, sino que la unidad tiene una prioridad ontológica, es decir, se posiciona en lo que Nicolás llama el modo de ser de la necesidad absoluta; la alteridad, en cambio, en el modo de ser propio de la contingencia. Por otro lado, subyacente a estos argumentos es dable indagar más a fondo que toda constatación de la alteridad lleva consigo el sello de la presencia de la eternidad, es decir, con aquello que trasciende toda mutabilidad, división o desigualdad. De este modo, la alteridad no es simple corrupción de la igualdad o de la conexión sino, dicho a modo de hipótesis, es signo transitorio del hecho trascendente de la unidad trinitaria y de su vínculo irreductible con ella.

En el nivel gnoseológico, por otro lado, Nicolás establece que el juicio de aprehensión consiste en el establecimiento de una proporción, que se constituye bajo la posibilidad de la conveniencia en algo uno y simultáneamente la alteridad. ${ }^{9} \mathrm{Si}$ bien la argumentación avanza para señalar que por la naturaleza del absoluto no hay proporción a lo infinito, de lo cual se afirma la ignorancia, se nos plantea la pregunta si esa distancia o negatividad es interpretable sólo como mera corrupción de la verdad, usando la misma expresión del nivel anterior, o si es posible articular una lectura coherente de ambos niveles. ¿Qué función cumple la alteridad en todo acto cognoscitivo?

A partir de este marco de ideas que hemos presentado de manera sintética, en el presente trabajo se pretende mostrar cómo desde la compleja noción de alteridad en el pensamiento cusano es posible reconocer el sello del principio unitrino en lo finito. Para lograr lo anterior, (i) se analiza la constatación negativa de la alteridad en la metafísica de la finitud vinculada a la contingencia, luego (ii) se describe la función articuladora que cumple el número en la comprensión de la finitud como multiplicidad creada por el primer principio de todo, Dios, toda vez que esta creación es un despliegue de la potencia del número divino. Finalmente, (iii) se analiza el lugar que tiene la alteridad en la argumentación trinitaria del libro primero de De docta ignorantia, con lo cual, mostraremos cómo la alteridad abre un espacio para una consideración del primer principio que, si bien excluye de sí toda alteridad, a la vez, funda la totalidad de lo otro.

natura; quare et connexio sicut unitas est aeterna, cum prior sit alteritate". 9 Cf. NICOLÁS DE CUSA, De docta ignorantia I (h. I, n. 3). 


\section{2) Alteridad y finitud ${ }^{10}$}

"Por lo tanto, la creatura tiene proveniente de Dios el que sea una, separada y conectada al universo, y cuanto más una tanto más semejante a Dios. Empero, el que su unidad sea en la pluralidad, la separación y la confusión, la conexión en la discordia, no lo tiene proveniente de Dios ni tampoco de alguna causa positiva, sino contingentemente" ${ }^{\text {"II }}$.

Tomado del libro II de De docta ignorantia, este pasaje nos remite a la noción utilizada por Nicolás para describir la índole propia de la alteridad de lo finito: la contingencia. En pocas palabras lo que se dice con esa noción es que la diferencia, como condición de lo diverso, no tiene una causa positiva, lo cual implica que la diferencia no se encuentra en la esencia de la cosa finita, sino que es algo extrínseca a ella. Nicolás apunta con la alteridad, por lo tanto, a un hecho irreductible o condición sin causa que se expresa en términos negativos, del no-ser.

En el tratado De ludo globi Nicolás vuelve a referirse al tema de la alteridad como manifestación del carácter perecedero de lo finito bajo la perspectiva de la creación:

"Dios crea todas las cosas, también las que son alterables, mutables y corruptibles; sin embargo, no crea la alteridad, no crea lo que muere, sino el ser. Que los seres se destruyan o se alteren no es producto del creador, sino de la contingencia"'2.

Con el vínculo a la idea de la creación, se excluye la perspectiva de la alteridad como un principio derivado o una potencia entendida como sustrato sobre el cual se añada la diferenciación o las determinaciones propias de la finitud. Es preciso señalar que el rechazo a considerar la

Io En esta parte seguiré parte de lo presentado en el IV Congreso Internacional Cusano de Latinoamérica. Nicolás de Cusa: Unidad en la Pluralidad, Buenos Aires, 24 al 27 de julio, 20I8, organizado por el Círculo de Estudios Cusanos de Buenos Aires y el Instituto de Filosofía de la Universidad de Buenos Aires, con la ponencia titulada: "La negatividad en la metafísica de la alteridad. Esbozo de una teología de la donación en el pensamiento cusano".

II Cfr. NICOLÁS DE CUSA, De docta ignorantia II (h. I, n. 99): "Habet igitur creatura a Deo, ut sit una, discreta et connexa universo et, quanto magis una, tanto Deo similior. Quod autem eius unitas est in pluralitate, discretio in confusione et connexio in discordantia, a Deo non habet neque ab aliqua causa positiva, sed contingenter". Nicolás de Cusa, Acerca de la docta ignorancia, Libro II: Lo máximo contracto o universo (edición bilingüe), J. MACHETTA, C. D’AMICO, S. MANZO (trads.), Biblos, Buenos Aires, 2009, p. 29.

I2 NICOLÁS DE CUSA, De ludo globi II (h. IX, n. 8I): "Omnia creat deus, etiam quae alterabilia et mutabilia et corruptibilia; tamen alteritatem et mutabilitatem corruptionemve non creat. Cum sit ipsa entitas, non creat interitum, sed esse. Quod autem intereant aut alterentur non habent a creante, sed sic contingit". 
alteridad como un principio está presente en las obras cusanas de distintos periodos, lo cual no significa que haya una caída en la inteligibilidad o una minusvaloración de la diferencia como condición de lo finito. Se trata, más bien, de reconocer que la alteridad queda de suyo excluida de los principios positivos de las creaturas, en la medida que no es posible acceder a un conocimiento de ellas a partir exclusivamente de la alteridad.

Si nos detenemos en un pasaje del libro I de De docta ignorancia, capítulo VII, encontramos una argumentación que vincula a la alteridad con el número en vistas a su relación con la unidad:

"Pues la alteridad es lo mismo que la mutabilidad. Pero todo lo que precede naturalmente a la mutabilidad, es inmutable, por lo cual es eterno. Empero la alteridad consta de lo uno y lo otro. Por lo cual la alteridad, como el número, es posterior a la unidad. Por lo tanto, la unidad es prioritaria, por naturaleza, a la alteridad y porque naturalmente la precede es unidad eterna"13.

Dejemos la explicación más detallada de la noción de número para más adelante, para decir únicamente que la característica relevante aquí es que el número es compuesto de unidad y alteridad y, por tanto, posterior a la unidad, lo cual permite establecer que la alteridad no es principio de la multiplicidad que el número despliega. La unidad, en efecto, precede tanto a la multiplicidad como a la alteridad, tal como el punto precede a la magnitud. Con estas reflexiones, el Cusano deja entrever que la alteridad no funciona como un principio a partir del cual la unidad se vuelve plural, sino que la alteridad sería más bien la multiplicidad en sí misma. Esta misma idea la encontramos presente en el Idiota de mente:

"Cuando tienes en cuenta que el número de la unidad se constituye por la multitud, y que la alteridad sigue, contingentemente, a la multiplicación, y adviertes la composición del número a partir de la unidad y de la alteridad, [...], y que la quididad de todo ha brotado para que sea el número de la mente divina, entonces, de alguna manera alcanzas cómo las esencias de las cosas son incorruptibles como la unidad, de la cual procede el número, que es la entidad, y cómo las cosas son de esta manera y de esta otra por la alteridad, la cual no es

I3 NICOLÁS DE CUSA, De docta ignorantia I, (h. I, n. I8): "Alteritas namque idem est quod mutabilitas; sed omne, quod mutabilitatem naturaliter praecedit, immutabile est; quare aeternum. Alteritas vero constat ex uno et altero; quare alteritas sicut numerus posterior est unitate. Unita ergo prior natura est alteritate et, quoniam eam naturaliter praecedit, est unitas aeterna". Nicolás de Cusa, Acerca de la docta ignorancia, Libro I: Lo máximo absoluto (edición bilingüe), J. MACHETTA, C. D’ AMICO (trads.), Biblos: Buenos Aires, 2009, p. 55. 
de la esencia del número, si no que sigue contingentemente la multiplicación de la unidad. Así, ciertamente, la alteridad no es de la esencia de ninguna cosa. Pues la alteridad pertenece a la muerte, porque es la división de la cual proviene la corrupción. De ahí que no es de la esencia de la cosa"14.

Siatendemos nuevamente alo referente al númeroy su composición de unidad y alteridad, el pasaje anterior nos muestra que lo que designa la alteridad es básicamente aquello que es el 2 en tanto otro o diverso del 3. Pero esta alteridad no coincide con la esencia del 2, como vimos, la alteridad se sigue de la multiplicación de la unidad sin causa positiva. La esencia del 2 y del 3 es en cada caso la unidad, el uno monádico, no obstante, el hecho de que se trate de una unidad discreta de 2 o de 3. Su esencia es siempre la misma, pues allí no hay alteridad. Una reflexión similar la encontramos en el ejemplo de la línea curva en el segundo capítulo del Libro II de De docta ignorancia donde se señala:

“[...] en cambio la línea curva, en cuanto línea, proviene de la infinita, en cuanto curva no proviene de la infinita sino que lo curvo es consecuencia de la finitud, porque por esto es curva: porque no es la máxima - pues si fuera máxima no sería curva [...]-, así ciertamente acontece para con las cosas, porque no pueden ser lo máximo de modo que sean disminuidas, diversas, distintas y lo que es similar, lo cual, ciertamente, no tiene causa"'

La curvatura de la línea, es decir, su finitud deriva de que ella no es la línea infinita. De modo semejante, que la cosa manifieste unidad se debe a Dios, la unidad absoluta desde la cual proviene. Ahora bien, que aquella unidad lo sea de unitas y alteritas ${ }^{16}$ es un evento de pura

I4 NICOLÁS DE CUSA, De mente (h.V, n. 96): "Quando attendis ex multitudine unitatis numerum constitui ac quod alteritas sequitur multiplicationem contingenter et advertis compositionem numeri ex unitate et alteritate, [...], ac quod quiditas rerum omnium exorta est, ut sit numerus divinae mentis, tunc aliqualiter attingis, quomodo essentiae rerum sunt incorruptibiles uti unitas, ex qua numerus, quae est entitas, et quomodo res sunt sic et sic ex alteritate, quae non est de essentia numeri, sed contingenter unitatis multiplicationem sequens. Ita quidem alteritas de nullius rei essentia est. Pertinet enim ad interitum alteritas, quia divisio est, ex qua corruptio. Hinc de essentia rei non est. Conspicis etiam, quomodo non est aliud numerus quam res numeratae. Ex quo habes inter mentem divinam et res non mediare numerum, qui habeat actuale esse, sed numerus rerum res sunt". NICOLÁS DE CUSA, Un ignorante discurre acerca de la mente. J. M. MACHETTA (trad.), Biblos: Buenos Aires, 2005, pp. 79-8I.

I5 NICOLÁS DE CUSA, De docta ignorantia II (h. I, n. 99): "Sicut enim linea infinita est rectitudo infinita, quae est causa omnis esse linealis, linea vero curva, in hoc quod linea, ab infinita est, in hoc quod curva, non ab infinita est, sed curvitas sequitur finitatem, quoniam ex eo curva, quia non maxima - si enim máxima esset, curva non esset [...] -: ita quidem contingit rebus, quoniam maximum esse non possunt, ut sint diminuta, altera, distincta et cetera huiusmodi, quae quidem causam non habent". NICOLÁS DE CUSA, Acerca de la docta ignorancia, Libro II: Lo máximo contracto o universo (edición bilingüe), J. MACHETTA, C. D' AMICO y S. MANZO (trads.), Biblos: Buenos Aires, 2009, p. 29.

I6 Cf. NICOLÁS DE CUSA, De coniecturis cap. IX (h. II, n. 37). 
contingencia. En este sentido, la contingencia no es otra cosa que expresión de la condición de no ser Dios, es decir, de una negatividad. Thomas McTighe lo resume de la siguiente manera: "A no es B debido a que A no es Dios, el absoluto" ${ }^{17}$. Tal negatividad es la que explica la alteridad, no al revés. La contingencia es, entonces, un estado ontológico, condición de ser esto y no lo otro, diferente de aquello; en otras palabras, como hemos señalado, la condición de ser múltiple. En atención a esto es que Nicolás observa que la alteridad no es un principio o causa positiva de aquella condición. En este punto, la diversidad concomitante a la participación queda excluida de la esencia o razón de la cosa misma. La contingencia no alude, por tanto, a una diversidad de esencias como explicación de la diferencia. Veamos esta idea en el ejemplo de la línea de dos y la de tres pies de la obra anteriormente citada:

“... por una parte, la razón de la línea en una y otra y en todas es una e igual; y por otra, hay diversidad entre la de dos pies y la de tres pies. De esta manera, una parece la razón de la línea de dos pies y otra la de tres pies. Ahora bien, es evidente que en la línea infinita no hay otra de dos pies y de tres pies. Y ella, la infinita, es razón de la finita. De donde una es la razón de ambas líneas, y la diversidad de cosas o bien de líneas no es por causa de la diversidad de la razón que es una sola, sino por accidente: porque no participan de la razón de modo igual"18.

La imposibilidad de participar de igual modo de la esencia única es otra manera de expresar esta condición de no ser el absoluto. Participar de la misma manera sería, en cualquier caso, ser igual a todas las otras líneas y, además, ser igual al absoluto. En pocas palabras no habría participación. La contingencia y la accidentalidad deben ser entendidas básicamente como una expresión de no ser el absoluto, de una diferencia radical, intrínseca con éste.

\section{3) Alteridad y conocimiento}

Hasta aquí hemos señalado que la noción cusana de alteridad, en el marco de la ontología, no expresa por sí misma una diferenciación de las esencias ni constituye una causa de la multiplicidad de lo finito en sí misma. Todas ellas son consideraciones que apuntan, más bien,

I7 Cf. T. McTIGHE, "Contingentia and Alteritas in Cusa's Metaphysics", American Catholic Philosophical Quarterly, Vol. 64 (1990), pp. 59.

I8 NICOLÁS DE CUSA, De docta ignorantia I (h. I, n. 48). NICOLÁS DE CUSA, Acerca de la docta ignorancia, Libro I: Lo máximo absoluto (edición bilingüe), J. M. MACHETTA, C. D' AMICO (trads.), Biblos: Buenos Aires, 2009, p. 85. 
a una negatividad concomitante a la diferenciación de lo finito. Ahora bien, estas ideas recogidas en los pasajes anteriores son presentadas por Nicolás con alusión directa a la noción de número. Efectivamente, dada la condición propia del número se observa cómo la diferenciación de lo finito es entendida como una multiplicidad articulada con otros principios relevantes de la metafísica y gnoseología cusanas. En este punto habría que decir que las alusiones al número no constituyen un elemento meramente ejemplificador, sino que, al poseer el estatuto de imagen, el número permite visualizar la unidad participada en la multiplicidad. En lo que sigue, aludiremos a este punto de manera más detallada, lo cual nos permitirá ahondar en el lugar de la alteridad en otros aspectos de la onto-gnoseología cusana.

En el capítulo VI del Idiota de mente, citado más arriba, se encuentra de manera sintética un buen contexto explicativo para la reflexión aludida tanto dentro del marco de la comprensión simbólica y racional del número de la mente divina y del carácter incorruptible de la esencia de las cosas. El postulado de un número de la mente divina, del cual el número matemático viene a ser imagen ${ }^{19}$, tiene resonancias pitagóricas claras. Si bien es cierto que la tradición platónica y pitagórica es parte del trasfondo de esta reflexión, es importante señalar, junto a Bocken ${ }^{20}$ y $\mathrm{D}^{\prime} \mathrm{Amico}^{2 \mathrm{~L}}$, que en el Cusano las nociones de número y unidad son desplazadas desde la realidad al ámbito de la actividad cognoscitiva humana. Este desplazamiento y su relación asociada a la noción de mens del capítulo en cuestión se vincula a temáticas ya presentados en su obra De coniecturis ${ }^{22}$. Uno de los temas decisivos es el paralelo entre la divina mens y la humana mens. Decisivo para la descripción de la multiplicidad, la cual no puede entenderse separada de la operación del propio espíritu, es decir, del modo de entender de la mens.

Antes de retomar el capítulo VI del De mente, convine detenernos en un pasaje de De docta ignorantia, que con justa razón $\mathrm{D}^{\prime}$ Amico considera cuasi "fundacional" ${ }^{23}$ en el pensamiento cusano, en el sentido que en él se vincula el uno simplísimo o principio primero (que no es el uno numérico) con la discreción de todo número:

I9 Cf. NICOLÁS DE CUSA, Idiota de mente (h. V, n. 88).

20 Cf. I. BOCKEN, "Die Zahl als Gründlage der Bedeutung bei Nikolaus von Kues". Mitteilungen und Forschungsbeiträge der Cusanus-Gesellschaft, Vol. 29 (2005), pp. 2I0-213; A. EISENKOPF, Zahl und Erkenntnis bei Nikolaus von Kues, Regensburg: Roderer-Verlag, 2007, pp. 9-I3.

2I C. D' AMICO, "Acerca del carácter irreductible de la mens humana en Nicolás de Cusa. Unidad y número”, Franciscanum. Revistas de Ciencias del Espíritu. 60 (2018), pp. 87-IO7.

22 Esta obra fue concluida por los años I44I-I442, unos 8-9 años antes de sus diálogos del Idiota.

23 C. D' AMICO, “Acerca del carácter irreductible de la mens humana...", p. 9I. 
"Y porque todas las cosas son de aquel modo mejor que el que pueden ser, entonces no puede haber pluralidad de entes sin el número. Pues suprimiendo el número cesan la distinción, el orden, la proporción, la armonía de las cosas y, además, la misma pluralidad de los entes [...], es claro que el ascenso del número es finito en acto y que tal número estará en potencia para otro. Y si en el descenso el número se comportara de la misma manera, que dado cualquier número pequeño en acto - puesto que, entonces, siempre podría darse, por sustracción, uno menor, como en el ascenso por adición se da uno mayor-, aun así sería lo mismo, porque no habría ninguna distinción de las cosas, y no se encontraría en los números ni orden, ni pluralidad, ni lo que excede ni lo excedido, aún más: no habría número"24.

Este texto podría llevarnos a pensar que Nicolás sostiene un pitagorismo clásico, en el sentido que Dios habría conformado la realidad de manera numérica. Sin embargo, esto no es propiamente lo que sostiene el Cusano ${ }^{25}$. En la progresión numerada tanto ascendente o descendente la unidad se explicita a sí misma de manera diversa. Los extremos absolutos, que superan esa progresión, es decir, lo máximo y lo mínimo absolutos, son concebidos como la unidad simplísima y coincidental o coincidencia de los opuestos. Ahora bien, el nexo para reconocer el lugar de la alteridad en este nivel lo encontramos en la referencia a la proporción como instrumento del conocimiento.

En De docta ignorantia Nicolás describe el conocimiento o juicio de aprehensión y aclara que consiste en el establecimiento de una comparación proporcional. En otras palabras, la comparación permite el tránsito desde lo conocido a lo desconocido gracias al instrumento de la proporción. En este punto el Cusano añade que la proporción, "[...] dado que implica la conveniencia en algo uno y simultáneamente la alteridad,

24 NICOLÁS DE CUSA, De docta ignorantia I (h. I, n. I3): "Et quoniam omnia sunt eo meliori modo, quo esse possunt, tunc sine numero pluralitas entium esse nequit; sublato enim numero cessant rerum discretio, ordo, proportio, harmonia atque ipsa entium pluralitas. [Quod si numerus ipse esset infinitus - quoniam tunc maximus actu, cum quo coincideret minimum -, pariter cessarent omnia praemissa. In idem enim redit numerum infinitum esse et minime esse.] Si igitur ascendendo in numeris devenitur actu ad maximum, quoniam finitus est numerus:non devenitur tamen ad maximum, quo maior esse non possit, quoniam hic foret infinitus. Quare manifestum est ascensum numeri esse finitum actu et illum in potentia fore ad alium. Et si in descensu pariter se numerus haberet, ut dato quocumque parvo numero actu, quod tunc per subtractionem semper dabilis esset minor sicut in ascensu per additionem maior, - adhuc idem; quoniam nulla rerum discretio foret, neque ordo neque pluralitas neque excedens et excessum in numeris reperiretur, immo non esset numerus".

25 Sobre el influjo de la tradición neopitagórica en Nicolás de Cusa ver: W. BEIERWALTES, "Einheit und Gleichheit. Eine Fragestellung im Platonismus von Chartres und ihre Rezeption durch Nicolaus Cusanus”, en W. BEIERWALTES, Denken des Einen. Studien zur neuplatonischen Philosophie und ihrer Wirkungsgeschichte, Klostermann: Frankfurt am Main, I985, pp. 368-384; R. HAUBST, Das Bild de Einen und Dreieinen Gottes in der Welt nach Nikolaus von Kues, Trierer Theologische Studien 4, Trier, 1952. pp. 209ss. 
no puede ser entendida sin el número. Por tanto, el número incluye todas las cosas proporcionables" ${ }^{26}$. Con lo dicho, es claro que las referencias al número no son una mera ejemplificación, sino que remiten al modo en que Nicolás articula un momento de su gnoseología y refieren a la alteridad como parte de su índole más propia. D'Amico resume esto diciendo que "cada número es idéntico a sí pero diverso de otro y esto es lo que lo vuelve comparable" ${ }^{27}$. En este sentido la multiplicidad, en la medida que es proporcionable parece provenir más bien del modo de operar de la mente humana en tanto ella la establece por medio de la proporción. Se clarifica, en ese sentido, la idea del desplazamiento a la actividad cognoscitiva humana, que señalamos más arriba, y cómo la alteridad se entiende como un constitutivo de dicha actividad.

Si retomamos el capítulo VI del De mente, la reflexión precedente permite considerar con más detalle la unidad y la multiplicidad en términos de la simplicidad y la composición del número. Para que esta consideración se entienda en su vínculo con la ontología cusana es preciso posicionar la visión intelectual en el plano de la unidad, cuestión que Nicolás señala al iniciar el capítulo: “... cuando afirmas "lo uno" abres la intelección acerca de cómo se hace la producción de las cosas...”28.

Primeramente, Nicolás llama simbólicamente número al primer principiado ${ }^{29}$ (primum principiatum) del cual se dicen dos consideraciones negativas: que (i) no es infinitamente simple y que (ii) no es compuesto de aquello de lo que él compone, vale decir, que se compone solo de sí mismo. La primera consideración es evidente pues es principiado y la segunda se entiende atendiendo a que, si se afirmara lo inverso, los componentes precederían por naturaleza al número, lo cual es contradictorio con la idea de que es el primer principiado. Esta particular posición del número tiene que ver, como ya hemos señalado, con la singularidad de su composición: él es compuesto y compuesto de sí mismo (del número par e impar se compone todo número). Por este motivo, Nicolás señala que la unidad del tres es compuesta de tres unidades, pero no separadamente, sino

26 NICOLÁS DE CUSA, De docta ignorantia I (h. I, n. 3): "Proportio vero cum convenientiam in aliquo uno simul et alteritatem dicat, absque numero intelligi nequit. Numerus ergo omnia proportionabilia includit". NICOLÁS DE CUSA, Acerca de la docta ignorancia, Libro I: Lo máximo absoluto (edición bilingüe), J. M. MACHETTA, C. D' AMICO (trads.), Biblos: Buenos Aires, 2009, p. $4 \mathrm{I}$.

27 C. D' AMICO, “Acerca del carácter irreductible de la mens humana...", p. 92.

28 NICOLÁS DE CUSA, De mente cap. VI (h. V, n. 88): "Et quando 'unum' dicis, intellectum aperis quomodo fit rerum productio [...]". NICOLÂS DE CUSA, Un ignorante discurre acerca de la mente. J. M. MACHETTA (trad.), Biblos: Buenos Aires, 2005, pp. 7I-73.

29 Cf. NICOLÁS DE CUSA, De mente cap. VI (h. V, n. 92). NICOLÁS DE CUSA, Un ignorante discurre acerca de la mente..., p. 75. 
que deben ser vistas como unidades que constituyen unidas el terciario. El número permite contemplar la unidad como una composición de unidad y multiplicidad y, a su vez, al ser sujeto de la proporción, permite articular la idea de la representación de la forma. Para elucidar aquello, Nicolás recurre al principio que define la proporción como el lugar de la forma (proportio est locus formae), pues "la proporción es como la aptitud de la superficie propia del espejo para el resplandor de la imagen, si la proporción no se mantiene deja de haber representación." ${ }^{\circ}$ Entonces, si la unidad infinita resplandece en la proporción más apta, esta debe ser el número dada su particular composición y naturaleza. En consecuencia, para Nicolás la pluralidad es en cierto modo multiplicidad numerada. Hay pluralidad puesto que hay una mens, o bien, en palabras del Cusano: "[...] así como respecto de Dios la pluralidad de las cosas es por la mente divina, así respecto de nosotros la pluralidad de las cosas es por nuestra mente. Pues únicamente la mente numera; quitada la mente no hay número que discierna"3.

El número divino, en consecuencia, debe entenderse como el ejemplar de las cosas, es decir, como la unidad en la mente increada que las une en una unidad anterior a toda pluralidad. Unidad que no debe confundirse con la unidad que une toda pluralidad concebible por la mente humana, pues ésta última es más bien semejanza de aquella mente increada. Como vemos, en esta meditación Nicolás liga dos niveles, el nivel del ser de la multiplicidad que debe ser, a su vez, comprendido sólo según el nivel o modo propio del entender. De ahí que el Nicolás llame al número ejemplar de la mens humana sobre la base del paralelismo establecido con la mens divina:

"De donde siendo el número el modo de entender, nada puede ser entendido sin él. Pues el número de nuestra mente es el ejemplar de las nociones por cuanto es la imagen del número divino, el cual es el ejemplar de las cosas. Y así como antes de toda pluralidad es la unidad, y la unidad que une es ésta: la mente increada en la cual todo es uno; y después del uno la pluralidad, explicación de la fuerza de esta unidad, la cual fuerza es la entidad de las cosas, la igualdad del ser y la conexión de entidad y de la igualdad; y ésta es la bendita trinidad. De esta manera en nuestra mente está la imagen de

30 NICOLÁS DE CUSA, De mente cap. VI (h. V, n. 92): "Est enim proportio quasi aptitudo superficiei specularis ad resplendentiam imaginis, qua non stante desinit repraesentatio". NICOLÁS DE CUSA, Un ignorante discurre acerca de la mente..., pp. 75.

3I NICOLÁS DE CUSA, De mente cap. VI (h. V, n. 93): "Unde sicut quoad deum rerum pluralitas est a mente divina, ita quoad nos rerum pluralitas est a nostra mente. Nam sola mens numerat; sublata mente numerus discretus non est". NICOLÁS DE CUSA, Un ignorante discurre acerca de la mente..., pp. 77. 
aquella trinidad divina"32.

La multiplicidad, como vemos en el pasaje anterior, es producto de la potencia entitativa de la mente divina que despliega la fuerza de su unidad en la explicatio. La condición de la explicatio, en ese sentido, va de la mano de la afirmación de la incorruptibilidad de las esencias de lo múltiple, pues éstas son semejanza de la unidad de la que procede el número divino.

Del lado de la mens humana, en el paso al nivel cognoscitivo, la alteridad queda en cierto modo interiorizada en la actividad de momento que el establecimiento de la proporción la supone como uno de sus aspectos constitutivos. En De conieturis esto queda plasmado en la descripción de la noción de conjetura. En el prólogo de esta obra remite a los hallazgos de De docta ignorantia, donde los había formulado en el lenguaje de la proporción, solo que en De coniecturis afirma que "[...] toda aserción humana de lo verdadero es conjetura" ${ }^{33}$. En tal sentido, la multiplicidad creada es modulada conjeturalmente por la mente humana en la medida que es asimilada en el momento productivo de su actividad dando como fruto el mundo "conjetural":

"Por lo tanto, la mente humana es forma conjetural del mundo tal como la divina del real. Por lo cual, así como aquella absoluta divina entidad es todo aquello que es, en todo lo que es, de la misma manera, también, la unidad de la mente humana es la entidad de sus conjeturas"34.

En este sentido, si hay una manera en que se conoce la unidad absoluta, este modo debe expresarse en términos conjeturales, en palabras de Nicolás: "es conocida la unidad de la inalcanzable verdad en

32 Nicolás de Cusa, De mente cap. VI (h. V, n. 95): "Unde cum numerus sit modus intelligendi, nihil sine eo intelligi potest. Numerus enim nostrae mentis cum sit imago numeri divini, qui est rerum exemplar, est exemplar notionum. Et sicut ante omnem pluralitatem est unitas, et haec est unitas uniens mens increata, in qua omnia unum, post unum pluralitas, explicatio virtutis illius unitatis, quae virtus est rerum entitas, essendi aequalitas et entitatis aequalitatisque conexio et haec trinitas benedicta, sic in nostra mente est illius trinitatis divinae imago. Nam mens nostra similiter est unitas uniens ante omnem pluralitatem per mentem conceptibilem, et post illam unitatem unientem omnem pluralitatem est pluralitas, quae est pluralitatis rerum imago sicut mens nostra divinae mentis imago". NICOLÂS DE CUSA, Un ignorante discurre acerca de la mente..., pp. 79.

33 NICOLÁS DE CUSA, De coniecturis, Prologus, (h. III, n. 2): "Quoniam autem in prioribus Doctae ignorantiae libellis multo quidem altius limpidiusque quam ego ipse nisu meo praecisionem veritatis inattingibilem intuitus es, consequens est omnem humanam veri positivam assertionem esse coniecturam." (la cursiva es mía).

34 NICOLÁS DE CUSA, De coniecturis (h III, n. 5): "Coniecturalis itaque mundi humana mens forma exstitit uti realis divina. Quapropter ut absoluta illa divina entitas est omne id quod est in quolibet quod est, ita et mentis humanae unitas est coniecturarum suarum entitas". 
la alteridad conjetural y también la misma conjetura de la alteridad en la simplísima unidad de la verdad."

\section{4) Alteridad y reflexión trinitaria}

El paralelismo de la mens divina y la mens humana, mencionado al finalizar el apartado anterior, ofreció un marco de fundamentación más amplio para la concepción cusana de la multiplicidad finita y su nota característica de la alteridad. La noción de número resultó clave para dicha caracterización. Ahora bien, este paralelismo considera, en el modo de la operación de la mens, además, la estructura trinitaria del primer principio del cual la mente humana es imagen. En lo que sigue, y teniendo en cuenta que la ontología cusana excluye la alteridad del primer principio, veremos el lugar que ocupa la noción de alteridad en la argumentación sobre la trinidad de la unidad absoluta en De docta ignorantia. Aun cuando este lugar es compartido con otras nociones relevantes dentro de la amplia reflexión desplegada por Nicolás, para nuestro propósito, servirá para reconocer cómo a partir de la alteridad de lo finito se plantea la articulación de dicho camino de argumentativo para elevar la reflexión metafísica hasta la estructura trinitaria del primer principio.

En el libro primero de De docta ignorantia Nicolás inaugura una tematización de la estructura trinitaria del primer principio de la mano de su manuductio simbólica aritmética y geométrica que pone de manifiesto la trinidad divina y que constituye una referencia fundamental para las especulaciones trinitarias de sus obras posteriores. Se trata de formas triádicas y ternarias de diverso orden que se vinculan con la doctrina trinitaria de la fe cristiana que reconoce en Dios tres hipóstasis. Estos caminos de desarrollo especulativo buscan desplegar una reflexión filosófica que asume una relación de determinación entre la identidad del absoluto y una diferenciación triádica del mismo.

En el capítulo VII del libro primero de esta obra, luego de mencionar a Pitágoras, a aquellos quienes adoraron la unidad entendiéndola como lo máximo, y de aludir a su tesis de que la unidad es trina, el Cusano desarrolla una serie de argumentos sobre la estructura trinitaria de la unidad absoluta. El primero avanza desde los constitutivos del mundo: alteritas y mutabilitas, para luego remontarse a su causa eterna e inmutable. La argumentación la encontramos resumida en el siguiente pasaje: 
"[...] nadie pone en duda que es eterno aquello que precede toda alteridad. Pues la alteridad es lo mismo que la mutabilidad. Pero todo lo que precede naturalmente a la mutabilidad, es inmutable, por lo cual es eterno. Empero, la alteridad consta de lo uno y lo otro. Por lo cual la alteridad, como el número, es posterior a la unidad. Por lo tanto, la unidad es prioritaria, por naturaleza, a la alteridad y porque naturalmente la precede es unidad eterna" ${ }^{35}$.

A continuación, Nicolás suma la reflexión en torno a la aequalitas para avanzar igualmente a la causa eterna. Si la desigualdad se compone de lo igual y lo excedente, entonces, siguiendo la vía de la sustracción, se hace evidente que la desigualdad se resuelve en la igualdad. En otras palabras, sustrayendo tanto de lo menor como de lo mayor se llega en cualquier caso a lo igual. De ahí que, sigue Nicolás, lo igual antecede a lo desigual ${ }^{36}$. A partir de esto concluye:

"Pero la desigualdad y la alteridad son simultáneas por naturaleza, pues donde hay desigualdad allí necesariamente hay alteridad y a la inversa. Pues entre, al menos dos cosas habrá alteridad. Una y otra, en verdad, respecto de una de las dos constituirán una duplicidad. Por lo cual, habrá desigualdad. En consecuencia, la alteridad y la desigualdad se darán por naturaleza simultáneamente, sobre todo siendo el binario la primera alteridad y la primera desigualdad. Pero queda establecido que la igualdad precede, por naturaleza, a la desigualdad, por lo cual también precede a la alteridad. En consecuencia, la igualdad es eterna" ${ }^{37}$.

Por último, el Cusano introduce la noción de divisio para completar su reflexión trinitaria en relación a la conexión eterna. Primero considera, que la unidad es conexión o causa de ella y, además, que el binario es como la primera división. En seguida, añade que entre dos causas de las cuales una es anterior a la otra, su efecto también lo sería respecto del efecto de aquella. Por lo tanto, puesto que la unidad es causa

35 NICOLÁ DE CUSA, De docta ignorantia I (h. I, n. I8): "Id, quod omnem alteritatem praecedit, aeternum esse nemo dubitat. Alteritas namque ídem est quod mutabilitas; sed omne, quod mutabilitatem naturaliter praecedit, immutabile est; quare aeternum. Alteritas vero constat ex uno et altero; quare alteritas sicut numerus posterior est unitate. Unitas ergo prior natura est alteritate et, quoniam eam naturaliter praecedit, est unitas aeterna". J. M. MACHETTA, C. D' AMICO, Nicolás de Cusa, Acerca de la docta..., p. 55-57.

36 Cf. NICOLÁ DE CUSA, De docta ignorantia I (h. I, n. 19).

37 NICOLÁ DE CUSA, De docta ignorantia I (h I, n I9): "Sed inaequalitas et alteritas simul sunt natura; ubi enim inaequalitas, ibidem necessario alteritas, et e converso. Inter duo namque ad minus erit alteritas. Illa vero ad unum illorum duplicitatem facient; quare erit inaequalitas. Alteritas ergo et inaequalitas simul erunt natura, praesertim cum binarius prima sit alteritas et prima inaequalitas. Sed probatum est aequalitatem praecedere natura inaequalitatem, quare et alteritatem; aequalitas ergo aeterna". J. M. MACHETTA, C. D' AMICO, Nicolás de Cusa, Acerca de la docta..., p. 57 . 
de la conexión y la división, por su parte, de la división, tenemos que la conexión es anterior por naturaleza a la división. Y culmina la reflexión observando:

"Pero la división y la alteridad son simultáneas por naturaleza. Por lo cual la conexión, también como la unidad, es eterna, por cuanto es anterior a la alteridad. Por lo tanto se ha probado que: porque la unidad es eterna, la igualdad es eterna, y también de modo similar la conexión es eterna. Pero no puede haber varios eternos. [...] de ahí que la unidad, la igualdad y la conexión son uno." 38

Como vemos Nicolás plantea tres caminos de argumentación cada uno ligado a la triple visión del mundo en sus notas características: alteritas, inaequalitas y divisio. Si nos atenemos a la argumentación misma, sin embargo, se observa que la alteridad funciona como un factor articulador en la media que permite un contrapunto en cada momento de la argumentación, que va desde la tríada del mundo creado a la tríada de las tres causas eterna: unitas, aequalitas y connexio.

Numerosos estudios han vinculado las argumentaciones precedentes con Agustín y, especialmente, con Thierry de Chartres. F. Resch ${ }^{39}$ ofreció recientemente una síntesis de la lectura de la tríada alteritas-inaequalitas-divisio a partir del Tractatus de sex dierum operibus como foco de interpretación de la tricausalidad fundamentadora de la tríada unitas-aequalitas-connexio ${ }^{40}$. Esa lectura pone de manifiesto justamente la correspondencia entre el lenguaje matemático simbólico y el contenido ontológico que está presente en la especulación cusana antes mencionada. Cada término de la tríada: alteridad, desigualdad y división constituyen tres caminos argumentativos que recogen la idea de tres causas inmanentes (causa material inmanente) que son creadas por las tres causas trascendentes, que Thierry identifica con las tres personas divinas. La consideración de la alteridad del mundo refleja la causalidad eficiente del mundo en la unitas. La desigualdad muestra la necesidad de la igualdad como causa formal trascendente del mundo y la división remite a la necesidad de la conexión como causa final trascendente. La causalidad eficiente es un momento necesario, pero no último ni suficientes para una aclaración del acto creador. La causa

38 NICOLÁ DE CUSA, De doc. ign. I, cap. VII (h I, n 2I).

39 F. RESCH, Triunitas. Die Trinitätsspekulation des Nikolaus von Kues, Aschendorff: Münster, 2014. Ver tb. C. RUSCONI, "Cusanus und Thierry von Chartres" en H. SCHWAETZER, K. ZEYER (hg.). Das europäische Erbe im Denken des Nikolaus von Kues. Geistesgeschichte als Geistesgegenwart, Aschendorff: Münster, 2008, pp. 285-302.

40 F. RESCH, Triunitas... pp. 65 ss 
eficiente corresponde a la iniciativa, la formal al momento reflexivo y la final al momento voluntario de dicho acto creativo. Las reflexiones que recogemos a partir de la argumentación articulada desde la alteridad se aclararan como tres aspectos del mundo creatural y que en Nicolás alcanzan una simbolización matemática en la alteridad como la prima alteritas o binario, la primera desigualdad y la primera división.

\section{5) Cierre}

Una buena manera de retomar la temática presentada al inicio de este trabajo es repasar el conocido pasaje de De visione Dei donde Nicolás sintetiza cada uno de los elementos problemáticos de la compleja noción de alteridad en el ámbito de lo finito:

"Tú me instruyes, Señor, de qué modo la alteridad, que no existe en ti, incluso no es en sí misma ni puede serlo. La alteridad, que no existe en ti, tampoco hace que una criatura sea diversa de otra, aunque una no sea la otra. El cielo no es la tierra, incluso aunque sea cierto que el cielo es el cielo y la tierra es la tierra. $\mathrm{Si}$, por tanto, quisiera buscar la alteridad, que no está en ti ni fuera de ti, ¿dónde podría encontrarle? Y si no existe, ¿cómo es que la tierra es una criatura diversa del cielo? Sin la alteridad, en efecto, esto no puede entenderse. Pero tú hablas dentro de mí, Señor, y me dices que no existe un principio positivo de la alteridad, y por eso la alteridad no existe. ¿Pues cómo la alteridad podría existir sin un principio, a no ser que ella misma fuese el principio y la infinitud? Pero la alteridad no es principio del ser. En efecto, la alteridad se nombra a partir del no-ser. Precisamente por el hecho de que una cosa no es otra, se dice que es otra. Por tanto, la alteridad no puede ser principio del ser, ya que se denomina a partir del no-ser, y tampoco tiene un principio de ser, puesto que procede del no-ser. La alteridad, por consiguiente, no es algo. La causa de que el cielo no sea la tierra se debe a que el cielo no es la misma infinitud, que abarca a todo ser"41.

Hemos mostrado que, abordando la terminología asociada a la alteritas, es posible reconocer un ámbito difuso e irreductible en la

4I NICOLÁS DE CUSA, De visione Dei (h. VI, n. 58): "Doces me, domine, quomodo alteritas, quae in te non est, etiam in se non est nec esse potest. Nec facit alteritas, quae in te non est, unam creaturam esse alteram ab alia, quamvis una non sit alia. Caelum enim non est terra, licet verum sit caelum esse caelum et terram terram. Si igitur quaesiero alteritatem, quae neque in te neque extra te est, ubi reperiam? Et si non est, quomodo terra est alia creatura quam caelum? Nam sine alteritate non potest hoc concipi. Sed loqueris in me, domine, et dicis alteritatis non esse positivum principium, et ita non est. Nam quomodo alteritas esset sine principio, nisi ipsa foret principium et infinitas? Non est autem principium essendi alteritas. Alteritas enim dicitur a non esse. Quod enim unum non est aliud, hinc dicitur alterum. Alteritas igitur non potest esse principium essendi, quia dicitur a non esse, neque habet principium essendi, cum sit a non esse. Non est igitur alteritas aliquid. Sed quod caelum non est terra, est, quia caelum non est infinitas ipsa, quae omne esse ambit." A. GONZÁLEZ, La visión de Dios, Eunsa: Navarra, 2007, pp. I07-I08. 
ontología cusana descrita en términos del no-ser. Sin embargo, como constitutivo propio de la finitud, la revisión de pasajes de obras cusanas nos permitió entender que la alteridad abre un espacio, justamente gracias a su negatividad, para articular la visión del mundo que no es autoexplicativo ni autosuficiente. Esta negatividad que abre la alteridad abre espacio para un camino argumentativo que, desde "abajo", permite elevarse hacia la estructura trinitaria del primer principio como tricausalidad. Ahora bien, hemos mostrado que estos hallazgos se logran, a su vez, en la medida que se observa la interiorización de la alteridad como momento constitutivo de la operación de la mens humana, gracias función paradigmática del número en la especulación cusana. 
